\title{
Biomimicry designs for passive optical solutions for nanoscale radiative cooling applications
}

Azadeh Didari, M. Pinar Mengüç

Azadeh Didari, M. Pinar Mengüç, "Biomimicry designs for passive optical solutions for nanoscale radiative cooling applications

," Proc. SPIE 10731, Nanostructured Thin Films XI, 107310C (7 September 2018); doi: 10.1117/12.2320504

Event: SPIE Nanoscience + Engineering, 2018, San Diego, California, United States 


\title{
Biomimicry designs for passive optical solutions for nanoscale radiative cooling applications
}

\author{
Azadeh Didari*, M. Pinar Mengüç*** \\ Center for Energy, Environment and Economy (CEEE), Ozyegin University, \\ Istanbul 34794, Turkey
}

\begin{abstract}
Inspired by the mechanism of the wings of Morpho butterfly, here we propose biomimicry designs which have the potential to be used for radiative cooling purposes. We numerically analyzed the spontaneous emission at near-field and determined radiative heat flux at nano-scale in order to investigate the impact of geometric variations and material selection in these systems. Our findings suggest that these metasurfaces which support phononic surface waves, can be used to tailor radiative heat transfer at nano-scale in the atmospheric transparency window $(8-13 \mu \mathrm{m})$ within the infrared regime.
\end{abstract}

Keywords: Nano-scale radiative cooling, phononic metasurfaces, biomimicry designs, Morpho butterfly, finite difference time domain method.

\section{INTRODUCTION}

Biologically inspired nanosystems can impact many scientific fields such as nanophotonics, nanomedicine, thermal therapy, energy harvesting and radiative cooling, among others ${ }^{1}$. Morpho butterflies with their blue or light-purple iridescence nature have inspired researchers from different backgrounds to investigate their structure ${ }^{2,3}$. While their structural based iridescent blue color serves the purpose of recognition in the male Morpho butterflies, the micro/nano structures within their wings and thorax have evolved over time in such a way that helps them survive intensive irradiation in tropical environments through an inhomogeneous temperature distribution ${ }^{4}$. In the recent years, different fabrication techniques ${ }^{5-10}$ have been developed to model the structures of Morpho butterflies with the intention to enhance the efficiency of solar cells ${ }^{9-11}$.

The inspiration for the present work came from Siddique et al. ${ }^{12}$ who investigated the nanostructures of the Morpho butterfly. They showed how the reflection spectrum can be controlled by the geometric design of the nanostructures, which is indeed the source of the strong blue iridescence of this butterfly's wings. A TEM image of the cross-section of the Morpho ground scale reveal what they call "Christmas-tree" ("pine-tree") like structure responsible for the wellknown blue iridescence ${ }^{8,12}$. Siddique et al. explored the source of broad reflection angle of these nanostructures and showed that among the most important design factors are the structural pattern of alternating branches within the structure and the height differences between neighbouring branches.

In the following sections, we discuss a number of structures inspired by wing structure of the Morpho butterfly which suggest that these highly reflective structures within the blue spectrum $(450-495 \mathrm{~nm})$ may have potential for attaining radiative cooling within the atmospheric window $(8-13 \mu \mathrm{m})$. Furthermore, we discuss possible extensions of this work that may help achieve significant enhancement of near-field radiative heat transfer in similar systems.

\section{RESULTS AND DISCUSSIONS}

In this section, we discuss various nano-structures inspired from the wings of Morpho didius butterfly. We outline their impact at near field, if another surface/geometry brought into the proximity of such biomimetic system, where the two

*Email: azadeh.didari@ozyegin.edu.tr

${ }^{* *}$ Email: pinar.menguc@ozyegin.edu.tr

Nanostructured Thin Films XI, edited by Akhlesh Lakhtakia, Tom G. Mackay, Proc. of SPIE Vol. 10731, $107310 \mathrm{C} \cdot(\mathrm{C}) 2018$ SPIE $\cdot$ CCC code: $0277-786 \mathrm{X} / 18 / \$ 18 \cdot$ doi: 10.1117/12.2320504 


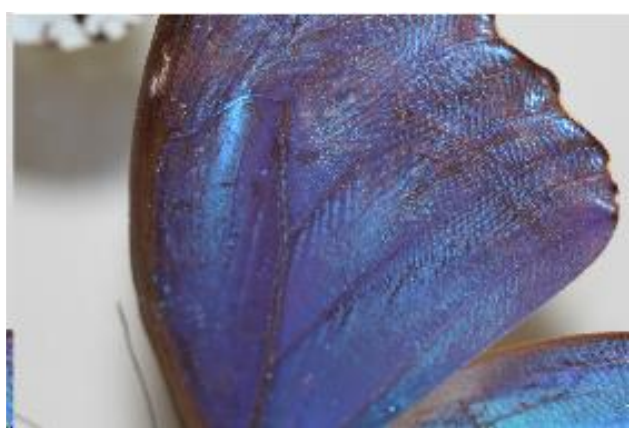

morpho didius butterfly wing

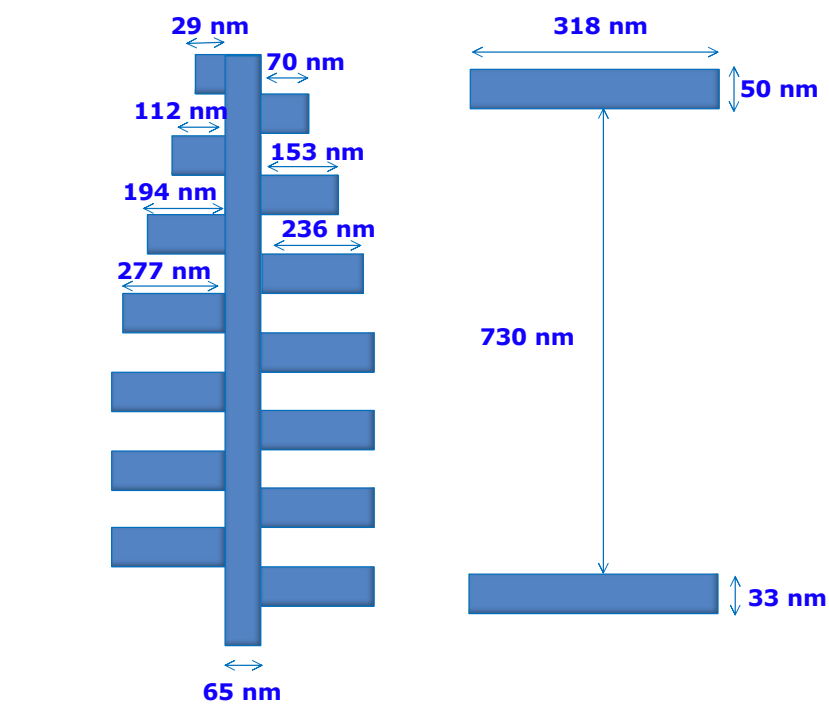

Original structure of morpho didus Benchmark

Figure 1. left: Morpho didius butterfly wing. Middle: the original structure of the wings of Morpho didius butterfly. Right: the benchmark scenario considered in this work.

media are separated by nano-scale vacuum gaps which are smaller or comparable proximity of the biomimetic system, where they are separated by nano-scale vacuum gap which is smaller or comparable to the wavelength of light in the surrounding environment. In these tree-like structures, the size of the branches, tree trunk, and interbranch separations are altered to achieve maximum enhancement of spectral and spatial radiative heat flux at nano-scale. The original sizes as well as the benchmark scenario considered for this work, are shown in Figure 1. The structures are made of $\mathrm{SiC}$ which is a phononic material which supports excitation of surface waves by surface phonon polaritons. Surface Phonon Polaritons $(\mathrm{SPhP})$ outperform their plasmonic counterparts within the infrared atmospheric transparency window due to their low loss nature within this range $(8-13 \mu \mathrm{m})$.

The first system we consider consists of two standing trees. Each tree is distanced from a standing thin film by a vacuum gap of $50 \mathrm{~nm}$ and is separated from the adjacent tree by $100 \mathrm{~nm}$. The schematics of this scenario are depicted in Figure 2 where the dimensions of the branches and various sizes are also given.

The near-field radiative heat flux at nano-scale is calculated by means of Dyadic Green's function. The full details of formulations can be found in ${ }^{13}$. The Ricker wavelet was used as the source of excitation ${ }^{14}$. The source was placed in the bottom of the left tree and the results were recorded for various spatial locations. However, we only report on the results calculated within the two trees, as indicated in Figure 2.

The numerical analysis is conducted by employing Near Field Radiative Transfer Finite Difference Time Domain (NF$R T$-FDTD) algorithm ${ }^{13}$. This algorithm is an in-house developed code designed specifically for nano-scale analysis of radiative heat flux at near-field. We performed 2D FDTD analysis with NF-RT-FDTD algorithm and used Drude-Lorentz dielectric function to model the frequency dependent optical characteristics of SiC. The grid size in both $X$ and $Z$ directions were set to $5 \mathrm{~nm}$ and the results of the performed convergence tests showed that convergence up to $0.5 \mathrm{~nm}$ mesh size can be achieved. The simulations were performed on a Hewlett-Packard HP-Z820 workstation, equipped with 2 CPUs of 10 physical and 20 logical cores each. System memory is $32 \mathrm{~Gb}$. This HP-Z820 is also fitted with a workstation-grade Nvidia GPU, the Quadro K4000 that has $3 \mathrm{~Gb}$ of very fast internal memory and 768 'CUDA' cores. On this system, the simulations took between 4-7 hours depending on a given configuration.

We first created a benchmark case for which analytical results can be obtained. This case is made of two thin SiC film separated from each other with a vacuum gap as depicted in Figure 1 (right). Then we focussed on combining various geometrical variations with the phononic characteristics of $\mathrm{SiC}$ and other metamaterials in order to examine the radiative 
heat flux within vacuum above the structure, particularly in the mid-infrared region $(8-13 \mu \mathrm{m})$ where we can use the surface phonon-polaritons modes of $\mathrm{SiC}$ to enhance the emissivity and hence radiative heat flux further.

When the system considered in Figure 2 is compared against the benchmark scenario, we observe that radiative heat flux at near-field depicts multiple spectral peaks that are up to three orders of magnitude higher than the benchmark scenario. The spectral enhancements are due to coupling of surface phonon polaritons within the branches of adjacent trees as well as the coupling of SPhPs due to the existence of the upper thin films. In the system considered, the upper thin layers are made of Lithium Fluoride (LiF) and the body of the trees are made of SiC. The optical constants of these materials are given in Table 1. Phononic materials such as $\mathrm{SiC}$ that have their emission peak within the infrared atmospheric window can be used to attain passive radiative cooling applications since they exhibit high emissivity within this window and low absorption outside of this window. In addition, phononic materials have their emission peak within the reststrahlen bandwidth which is defined between longitudinal $\left(\omega_{L O}\right)$ and optical $\left(\omega_{T O}\right)$ frequencies of a given material. Among phononic materials, LiF has one of the highest reststrahlen bandwidths. For this reason, it is possible to achieve spectral tunability over a large bandwidth with LiF.

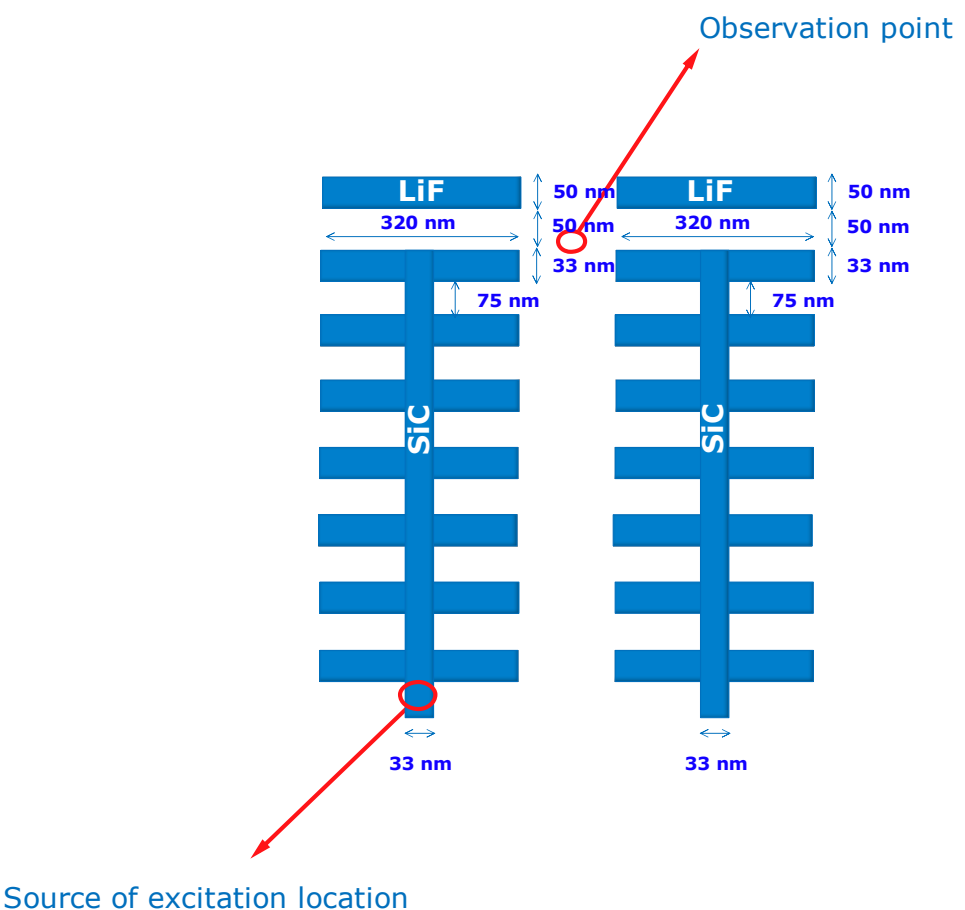

Figure 2. The schematics of the first proposed system. The separation distance between the two adjacent trees is $100 \mathrm{~nm}$. 
Table 1. Optical constants of materials.

\begin{tabular}{|c|c|c|c|c|}
\hline & $\omega_{L O}$ & $\omega_{T O}$ & $\Gamma$ & $\varepsilon_{\infty}$ \\
\hline $\mathrm{LiF}$ & $12.3 \times 10^{13} \mathrm{rad} / \mathrm{s}$ & $6.72 \times 10^{13} \mathrm{rad} / \mathrm{s}$ & $1.5 \times 10^{11} \mathrm{~s}^{-1}$ & 2.63 \\
\hline $\mathrm{SiC}$ & $1.82 \times 10^{14} \mathrm{rad} / \mathrm{s}$ & $1.49 \times 10^{14} \mathrm{rad} / \mathrm{s}$ & $8.96 \times 10^{11} \mathrm{~s}^{-1}$ & 6.7 \\
\hline
\end{tabular}

We aim to observe the impact of combining geometric variations and material characteristics of these two materials in the scenario examined in the Figure 2. Our findings suggest that as a result of using $\mathrm{LiF}$ as the material of the upper thin layers, the spectral peaks are shifted towards the outside of the first atmospheric window $\left(1.45 \times 10^{14} \mathrm{rad} / \mathrm{s}\right.$ $\left.2.35 \times 10^{14} \mathrm{rad} / \mathrm{s}\right)$ and towards the second atmospheric window $\left(1.17 \times 10^{14} \mathrm{rad} / \mathrm{s}-7.85 \times 10^{13} \mathrm{rad} / \mathrm{s}\right)$.

In the next cases presented in Figures 4 and 6, we only use $\mathrm{SiC}$ and observe that the additional spectral peaks occur within the first atmospheric window with variations which can be associated with the geometric characteristics of these systems. It is possible to increase the magnitude of the radiative heat flux by modifying the spatial location of the source of excitation, the size and dimensions of the systems and the materials selected.

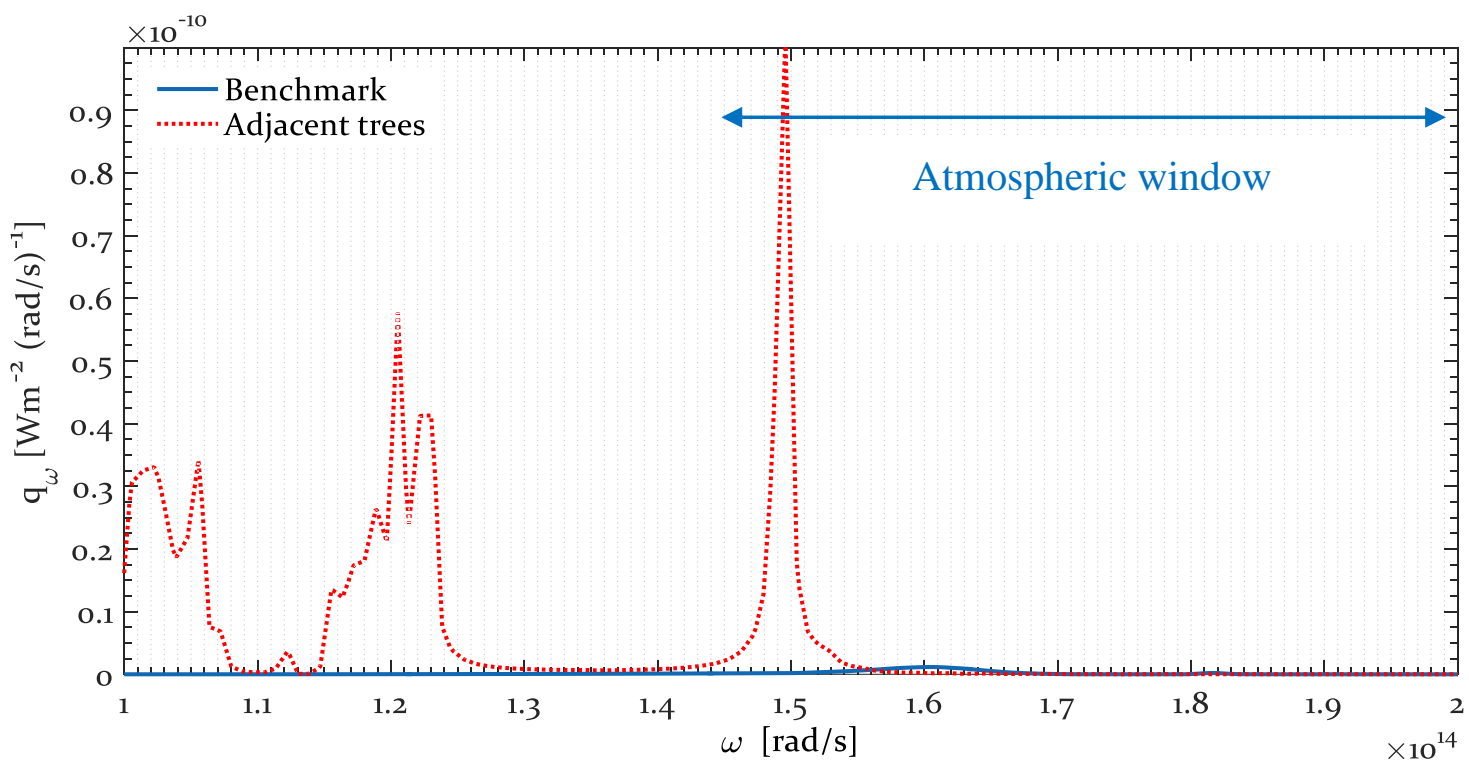

Figure 3. The near-field radiative heat flux for the geometric system depicted in Figure 2. 


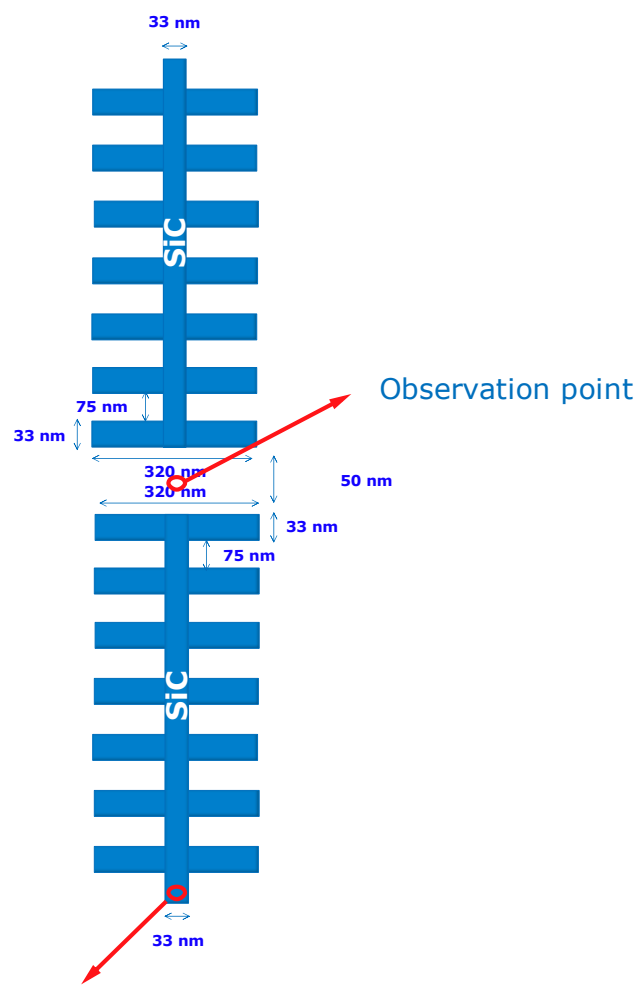

Source of excitation location

Figure 4. The schematics of the second proposed system.

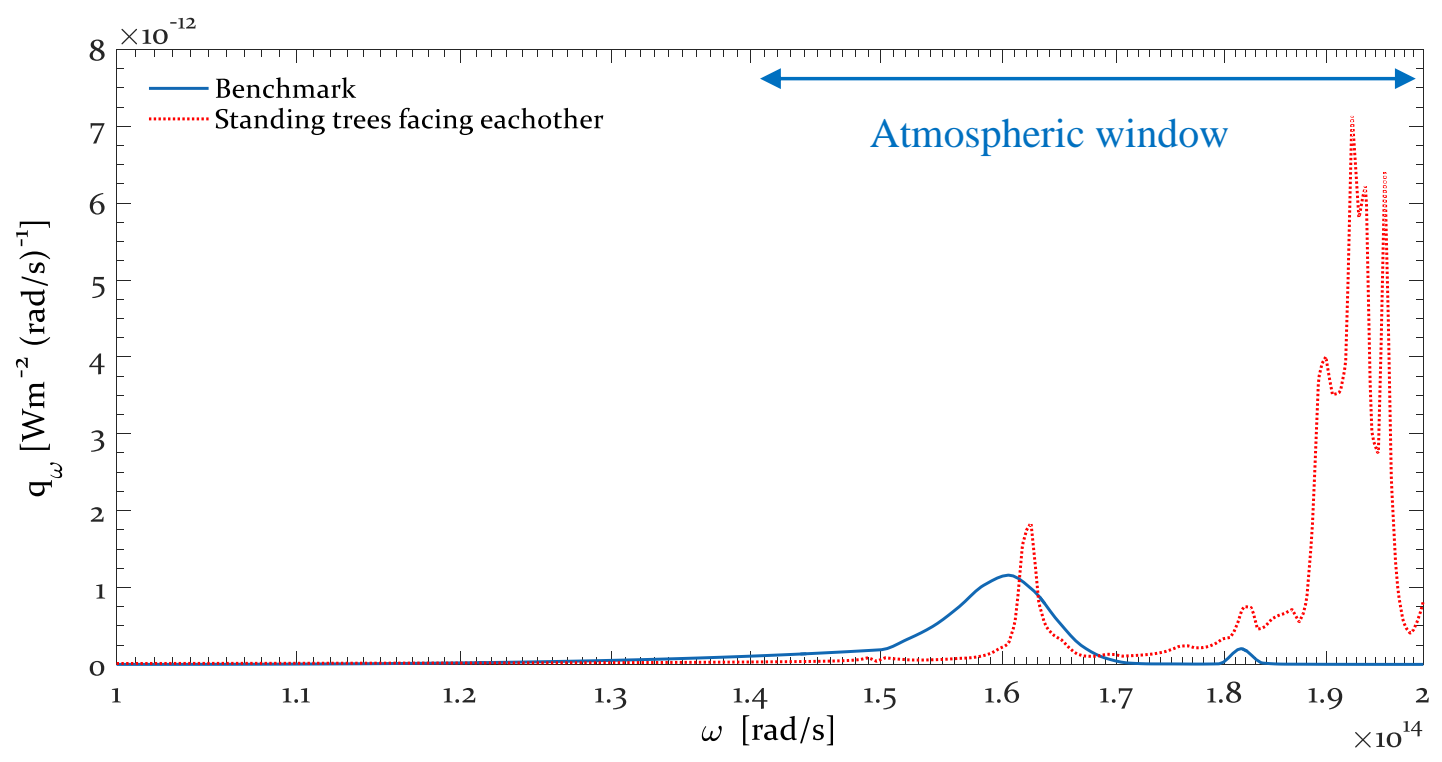

Figure 5. The near-field radiative heat flux for the geometric system depicted in Figure 4.

Proc. of SPIE Vol. 10731 107310C-5 


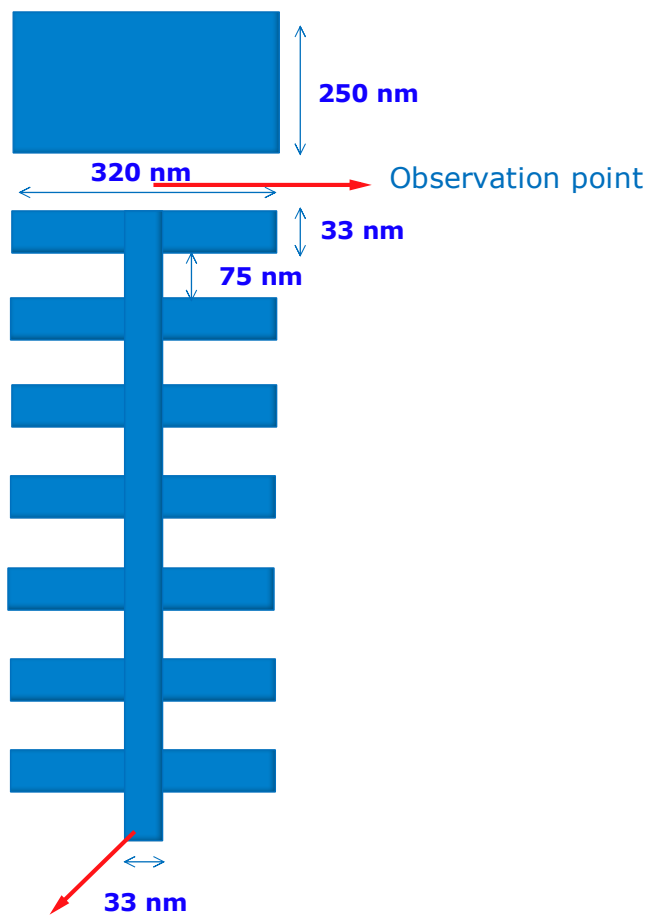

Source of excitation location

Figure 6. The schematics of the third proposed system.

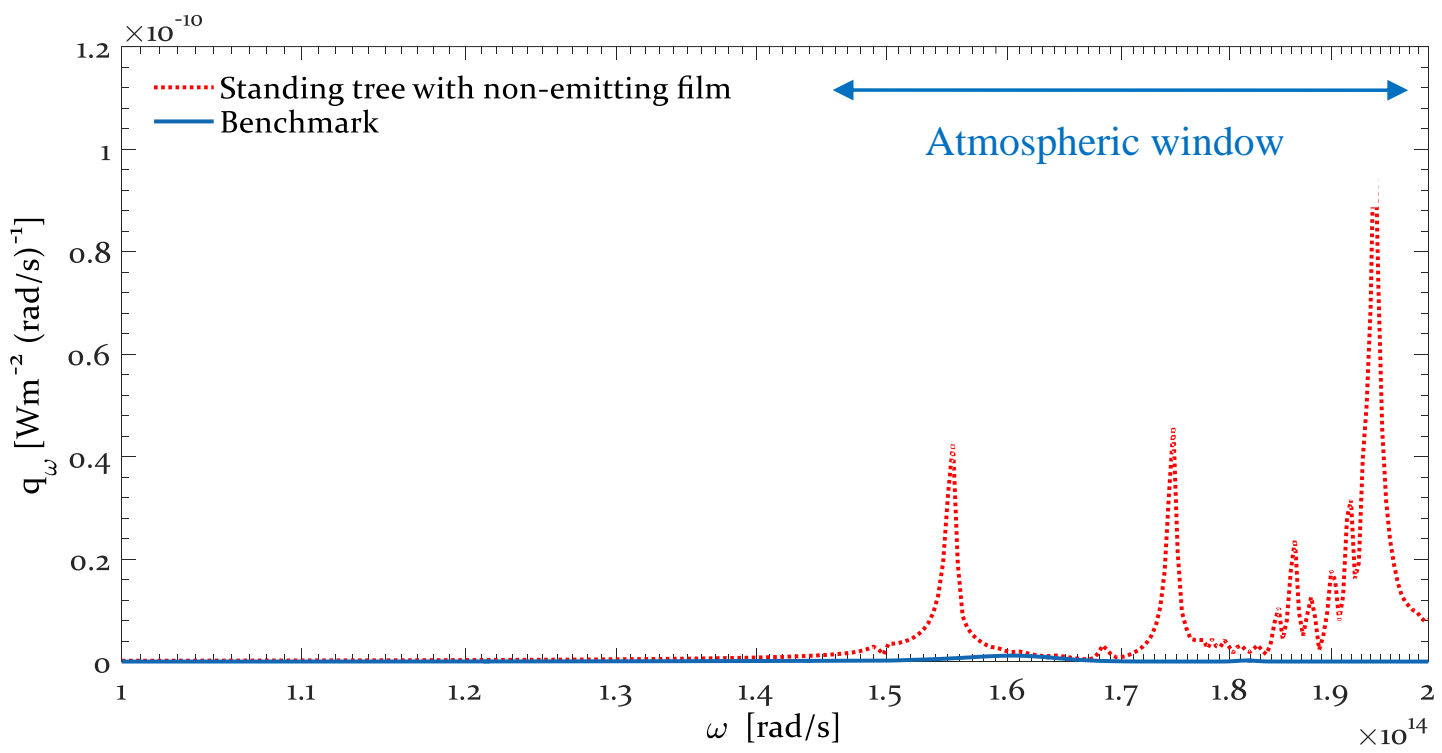

Figure 7. The near-field radiative heat flux for the geometric system depicted in Figure 6. 


\section{CONCLUSIONS}

We have conducted an FDTD analysis of biologically inspired designer nanostructures by using NF-RT-FDTD numerical algorithm. We investigated the near-field heat flux profiles of the tree-like structures which are inspired by the structure of Morpho butterflies. The results suggest that when considering a phononic metamaterial such as $\mathrm{SiC}$ in these structures, within the radiative cooling window, heat flux profiles experience enhanced values with multiple peaks occurring at different wavelengths. However, outside this window, they are strongly suppressed.

This behavior suggests a potential application of these structures for radiative cooling applications for thermal management of electronic sensors. We believe it is possible to enhance the near-field emission and heat flux in these systems by tailoring their design characteristics based on the selection of different materials and geometries. For instance, increasing the number of adjacent trees in the presence of an upper absorbing thin film may be employed to increase the near-field radiative heat transfer significantly for energy harvesting applications in the proposed structures. While we focused on only the near-field radiative transfer in this work, in the future, we will also explore the far-field thermal radiation.

\section{REFERENCES}

[1] Martín-Palma, R. J. \& Lakhtakia, A. Bioreplication for optical applications. MRS Commun. 8, 220225 (2018).

[2] Singamaneni, S. ; Introduction: Bioinspired and Biomimetic Materials. Chem. Rev. 117, 12581-12583 (2017).

[3] Shi, N. N. et al. Keeping cool: Enhanced optical reflection and radiative heat dissipation in Saharan silver ants. Science (80-. ). 349, 298-301 (2015).

[4] Chia Tsai, Cheng; Nan Shi, Norman; Ren, Crystal; Pelaez, Julianne ; D. Bernard, Gary; Yu, Nanfang; Pierce, N. Butterflies regulate wing temperatures using radiative cooling. in (SPIE, 2017).

[5] Saito, A., Ishikawa, Y., Miyamura, Y., Akai-Kasaya, M. \& Kuwahara, Y. Optimization of reproduced Morpho-blue coloration - art. no. 676706. in PHOTONIC CRYSTALS AND PHOTONIC CRYSTAL FIBERS FOR SENSING APPLICATIONS III 6767, 76706 (2007).

[6] Asano, M; Kuroda T; Shimizu, S; Sakihara, A; Kumazawa, K; Tabata, H. Morphotex fiber. (2001).

[7] Potyrailo, R. A. et al. Morpho butterfly wing scales demonstrate highly selective vapour response. Nat. Photonics 1, 123-128 (2007).

[8] Pris, A. D. et al. Towards high-speed imaging of infrared photons with bio-inspired nanoarchitectures. Nat. Photonics 6, 195-200 (2012).

[9] Lou, S., Guo, X., Fan, T. \& Zhang, D. Butterflies: Inspiration for solar cells and sunlight watersplitting catalysts. Energy and Environmental Science 5, 9195-9216 (2012).

[10] Heilman, B. D. \& Miaoulis, I. N. Insect thin films as solar collectors. Appl. Opt. 33, 6642-6647 (1994).

[11] Zhang, W. et al. Novel photoanode structure templated from butterfly wing scales. Chem. Mater. 21, 33-40 (2009).

[12] Siddique, R. H., Diewald, S., Leuthold, J. \& Hölscher, H. Theoretical and experimental analysis of the structural pattern responsible for the iridescence of Morpho butterflies. Opt. Express 21, 14351 (2013).

[13] Didari, A. \& Pinar Mengüç, M. A design tool for direct and non-stochastic calculations of near-field radiative transfer in complex structures: The NF-RT-FDTD algorithm. J. Quant. Spectrosc. Radiat. Transf. 197, (2017).

[14] Schneider, J. B. Understanding the Finite-Difference Time-Domain Method. Self Published (2013).

[15] Francoeur, M., Mengü, M. P. \& Vaillon, R. Near-field radiative heat transfer enhancement via surface phonon polaritons coupling in thin films. Appl. Phys. Lett. 93, (2008). 